Historic, archived document

Do not assume content reflects current scientific knowledge, policies, or practices. 



\section{BOX LIST NUMBER ONE MARCH 22, 1926}

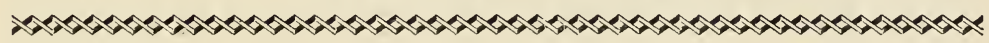

\section{CHASE NURSERY COMPANY}

(INCORPORATED)

CHASE, ALABAMA

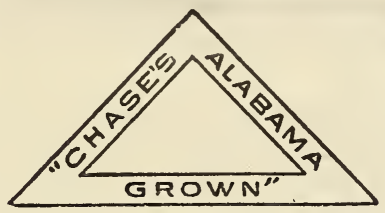

\section{BOXED LOTS FROM COLD STORAGE}

Orders for St. Louis Boxes Should be sent to

CHASE NURSERY COMPANY

c/o. BOOTH COLD STORAGE

Main \& Ashley Sts.

St. Louis, Mo.

St. Louis Telephones, Olive 5620

$\begin{array}{lll}\text { (Ask for Chase) } \quad \text { "6 } & 5621 \\ & \text { "6 } & 5622\end{array}$

Orders for Rochester Boxes Should Be Sent to

ROBERT C. CHASE

HOTEL SENECA

ROCHESTER, NEW YORK

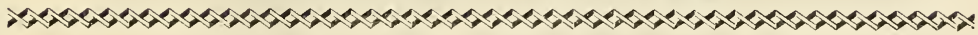

REMEMBER :

We guarantee condition of every Boxed Lot from Cold Storage.

Your planting season is prolonged several weeks by this service. 


\section{To The Trade:}

This Bulletin covers Boxed Lots in cold storage at St. Louis and Boxed Lots in cold storage at Rochester, New York. Our offices in St. Louis and Rochester are now open for the dispatch of business. "Quick action" is the motto of both offices.

A code word is given for each boxed lot, and your orders by wire or letter will get the goods rolling RIGHT NOW-or will reserve for later shipment as desired.

WEIGHT OF BOXES is given so that you can figure on express shipments intelligently.

Do you realize that Cold Storage is a very valuable thing to the Nurserymen? These Boxed Lots are kept in a uniform temperature of 33 degrees. It retards the flow of sap and thereby prolongs the planting season for weeks. The landscape planter in Florida even can draw on these Boxed Lots as late as April 15th and receive stock in perfect condition. Bear this in mind if you are now wondering how you can handle all your planting work this spring. Book the order, let us reserve the boxes for shipment after the middle of April if desired.

TERMS and Conditions of Sale as per our regular Trade List; cash with order where purchaser's credit is unknown to us.

ORDERS booked in rotation as received.

Always name second choice if possible.

Shipments will be made when desired.

RUSH SERVICE on Boxed Lots is easy; no packing to do, simply grab a box and get it on the express wagon or dray. Henry Chase is in St. Louis and Robert Chase in Rochester to handle the shipping. If possible get your freight orders to us before 3:00 p.m., for shipment that day. Express orders will go out on night trains, if received by $4: 30$ p.m.

Orders for Boxes In St Louis Should Be Sent To

CHASE NURSERY COMPANY

c/o. BOOTH COLD STORAGE

Main \& Ashley Sts.

ST. LOUIS, MO.

Orders for Boxes In Rochester Shoulä Be Sent To ROBERT C. CHASE

Hotel Seneca

ROCHESTER, N. Y. 


\section{BOXED LOTS-HOW TO ORDER}

A code word is given for each lot, also the approximate weight of each box. If, for instance, you want one box Privet, code HOOK, and one box Spirea VanHoutte, code SOIL, your wire or letter should read, "Freight (or Express) one each, HOOK, SOIL."

Address All Orders for Any of These St. Louis Boxed Lots To:

\section{CHASE NURSERY COMPANY}

c/o. Booth Cold Storage

Main \& Ashley Sts.

ST. LOUIS, MISSOURI

CODE WORD

PRIVET-IN ST. LOUIS

PRICE

PER BOX

$\$ 30.00$

Hoard-1 Box (770 lbs.)

Containing 350 Amoor River North, Assorted grades, as follows:

130 2-3 ft., 4 or more canes

130 18-24 in., 3 or more canes

90 12-18 in., 3 or more canes

Hobby-2 Boxes (700 lbs.)

Each containing 1500 Amoor River North, 2-3 ft., 2 and 3 canes.

Holiday -2 Boxes (520 lbs.)

Each containing 1850 Amoor River North, 18-24 in., 2 and 3 canes.

Hollow-2 Boxes (600 lbs.)

75.00

Each containing 1850 Amoor River North, 12-18 in., 3 or more canes.

Home-4 Boxes (460 lbs.)

Each containing 2500 Amoor River North, 12-18 in., 2 canes.

Honest-4 Boxes (380 lbs.)

Each containing 2500 Amoor River North, 6-12 in., branched.

Honey -4 Boxes (250 lbs.)

Each containing 2500 Amoor River North, transplanting.

Hook-8 Boxes (165 lbs.)

Each containing 1000 California, 6-12 in., branched.

Horn-2 Boxes (375 lbs.)

Each containing 1000 Vulgaris, $12-18$ in., 3 or more canes.

\section{ROSES-IN ST. LOUIS}

Rule-2 Boxes (160 lbs.)

Each containing 100 Assorted H.P. No. 1, as follows:

20 Frau Karl Druschki, Grafted

30 Gen. Jack, Budded

10 Hugh Dickson, Budded
20 Marshall P. Wilder, Budded

20 Paul Neyron, Budded

Rump-2 Boxes (155 lbs.)

Each containing 100 Assorted Climbers No. 1, Own Root, except as noted:

10 American Pillar

10 Christine Wright

10 Cli. Baby Rambler

10 Cli. Orleans
20 Crimson Rambler

10 Excelsa

10 Paul's Scarlet (Budded)

20 Seven Sisters 


\section{CODE WORD}

Each containing 100 Crimson Rambler No. 1, Own Root.

Rut-2 Boxes (370 lbs.)

Each containing 110 Rosa Rugosa Rubra, 2 yr., 2-3 ft., well br.

Rusty-2 Boxes (360 lbs.)

Each containing 200 Rosa Rugosa Rubra, 18-24 in., 2 yr., well br.

Rye-1 Box (335 lbs.)

Containing 300 Rosa Rugosa Rubra, 12-18 in., 2 yr., well br.

\section{SHRUBS-IN ST. LOUIS}

Slam-3 Boxes (515 lbs.)

Each containing 330 Assorted Shrubs, 2 yr., 2-3 ft., as follows: 20 Deutzia Pr. of Rochester

30 Lon. Fragrantissima

10 Lon. Tart Alba

20 Jasminum Nudifiorum

30 Phil. Nivalis

10 Lon. Morrowii

40 Spirea Prunifolia

40 Weigela Stelzneri

50 Phil. Grandiflorus

50 Spirea Arguta

30 Symphoricarpos Vulgaris

Slate-3 Boxes (235 lbs.)

Each containing 200 Assorted Shrubs, 2 yr., 18-24 in., as follows:

50 Lonicera Fragrantissima

50 Deutzia Crenata Rosea

50 Phil. Grandiflorus

20 Jasminum Nudiflorum

30 Lonicera Morrowii

Slice-1 Box (470 lbs.).

Containing 295 Assorted Shrubs, 2 yr., 18-24 in., as follows: 100 Spirea Reevesiana fl. pl. 55 Weigela Variegated 50 Spirea Callosa alba 90 Viburnum Dentatum

Sled-1 Box (290 lbs.)

Containing 420 Assorted Shrubs, 2 yr., 12-18 in., except as noted: 40 Deutzia Crenata 200 Spirea Arguta

30 Deutzia Gracilis (8-12 in.) 75 Symphoricarpos Mollis

50 Hydrangea P. G. 25 Weigela Candida

Slug-4 Boxes (450 lbs.)

Each containing 200 Berberis Thunbergi, 24-30 in., 3 year.

Smack-3 Boxes (700 lbs.).

Each containing 500 Berberis Thunbergi, 18-24 in., 3 year.

Smirk-1 Box (465 lbs.)

380 Berberis Thunbergi, 15-18 in., 3 year.

Snag-2 Boxes (230 Ibs.)

Each containing 300 Deutzia Crenata, 18-24 in., 2 year.

Sneer-1 Box (360 lbs.).

Containing 135 Upright Honeysuckle, 2 yr., $2-3$ ft., as follows: 75 Ruprechtiana

60 Tatarica Rubra

Soap-1 Box (250 lbs.)

Containing 200 Assorted Philadelphus, 2 yr., 2-3 ft., as follows:

90 Grandiflorus

80 Nivalis

30 Speciosissimus

Sock-1 Box (350 lbs.)

Containing 160 Spirea callosa aiba, 2 yr., 15-18 in.

Sofa-1 Box (270 lbs.)

Containing 260 Spirea callosa alba, 12-15 in., 2 yr.

Soil-3 Boxes (320 lbs.)

Each containing 500 Spirea VanHoutte, 2 yr., 18-24 in.

Solid-3 Boxes (225 lbs.)

Each containing 500 Spirea VanHoutte, 2 yr., 12-18 in. 


\section{IN COLD STORAGE AT ROCHESTER, N.Y.}

\section{BOXED LOTS-HOW TO ORDER}

A code word is given for each lot also approximate weight of each box. If, for instance, you want one box Crimson Rambler XX, code word RACK, and two boxes. Crimson Rambler No. 1, code word RANCID, your wire (or letter) should read, "Freight (or Express) one RACK, two RANCID."

Address All Orders for Rochester Boxed Lots To

\section{ROBERT C. CHASE}

\section{Hotel Seneca}

HOCHESTER, N. Y.

\section{ROSES, XX GRADE-IN ROCHESTER}

These carry about 30 inches wood same as Number One but are extra heavy canes. Probably not worth any more than the Number One grade, but taken out to make grade more uniform.

PRICE CODE WORD

PER BOX

Rabid-1 Box (245 lbs.) $\$ 56.00$

Containing 160 Assorted as follows:

85 Gen. Jack, Buds

20 Paul's Scarlet Climber, Buds 10 Prince Camille, Buds

20 Mary Wallace, Own Root 25 Crimson Rambler, Own Root

Race-1 Box (360 lbs.). 37.50

Containing 150 Assorted, Own Root, as follows:

50 American Pillar

70 Cli. Baby Rambler

20 Cli. Cecil Brunner

$10 \mathrm{Cli}$. Orleans

Rack-2 Boxes (560 lbs.)

Each containing 200 Crimson Rambler, Own Root.

Racket-1 Box (550 lbs.)

Containing 200 J. B. Clarke, Budded.

Radiate-1 Box (510 lbs.)

Containing 250 Paul Neyron, Budded.

Radical-1 Box (290 lbs.)

Containing 100 Philadelphia Rambler, Own Root.

Radish-1 Box (275 lbs.)

Containing 110 Tausendschon, Own Root.

\section{ROSES-NUMBER ONE GRADE-IN ROCHESTER}

Carrying 18 to 30 inches wood according to habit of growth, with at least three strong canes.

Containing 150 Assorted, Own Root, as follows: 135 Anna Mueller (Pink Baby)

15 Baby Echo

Raft-1 Box (240 lbs.)

Containing 160 Assorted, Own Root, as follows:

30 Aviator Bleriot

30 Cli. Cecil Brunner

10 Queen of Prairie

10 Rosarie
$10 \mathrm{Cli}$. Clothilde Soupert

40 Frau. Octavia Hesse

10 Fortunes D'ble Yellow

20 Tausendschon 
ROSES, NUMBER ONE GRADE, IN ROCHESTER-Continued

\section{CODE WORD}

PER BOX

Rage-1 Box (175 lbs.)

$\$ 35.00$

Containing 100 Assorted Buds, as follows:
$20 \mathrm{His}$ Majesty
10 Conrad F. Meyer
25 Prince Camille
45 J. B. Clarke

Ragged-1 Box (150 lbs.)

Containing 145 Assorted, Own Root, as follows:

50.75

10 Hugonis

85 Magna Charta

50 New Century

Rain-1 Box (270 lbs.)

Containing 230 Own Root, as follows:

100 Christine Wright
80 Graf Zeppelin

Raise-2 Boxes (285 lbs.).

Each containing 250 American Pillar, Own Root.

Rake-1 Box (215 lbs.)

Containing 110 Bess Lovett Buds.

Rally-2 Boxes (275 lbs)

Each containing 250 Cli. Baby Rambler, Own Root.

Ranch-1 Box (375 lbs.)

Containing $280 \mathrm{Cli}$. Orleans, Own Root.

Rancid-2 Boxes (300 lbs.).

Each containing 250 Crimson Rambler, Own Root.

Random-2 Boxes (285 lbs.).

Each containing 250 Excelsa, Own Root.

Range-1 Box (160 lbs.)

Containing 90 Excelsa, Own Root.

Rank-1 Box (230 lbs.)

Containing 150 Frau Karl Druschki, Grafted.

62.50

33.00

62.50

70.00

62.50

62.50

22.50

52.50

Rap-2 Boxes (285 lbs.)

Each containing 240 Gen. Jack, Buds.

Rapid-1 Box (325 lbs.)

Containing 300 Gen. Jack, Buds.

Rapture-2 Boxes (260 lbs.).

62.50

Each containing 250 Graf Zeppelin, Own Root.

Rarity-2 Boxes (335 lbs.).

Each containing 200 J. B. Clarke, Buds.

70.00

Rascal-1 Box (255 lbs.).

63.00

Containing 180 Marshall P. Wilder, Buds.

Rash-1 Box (205 lbs.).

Containing 160 Mary Lovett, Buds.

Rat-2 Boxes (310 lbs.)

Each containing 250 Paul Neyron, Buds.

Ratify-1 Box (160 lbs.)

Containing 170 Paul Neyron, Buds.

Ration-2 Boxes (200 lbs.)

Each containing 160 Paul's Scarlet Climber, Buds.

Rattle-2 Boxes (300 lbs.)

Each containing 250 Philadelphia Rambler, Own Root. 


\section{IN COLD STORAGE AT ROCHESTER, N. Y.}

ROSES, NUMBER ONE GRADE, IN ROCHESTER-Continued

CODE WORD

PER BOX

Rave-1 Box (190 lbs.) $\$ 30.00$

Containing 100 Purity, Buds.

(Said to be an improvement on SILVER MOON.)

Ray-2 Boxes (210 lbs.)

Each containing 250 Seven Sisters, Own Root.

Razor-2 Boxes (220 lbs.).

Each containing 195 Seven Sisters, Own Root.

\section{ROSES, MEDIUM GRADE-IN ROCHESTER}

Carrying about eighteen inches wood with average of three canes. A real good grade.

Rim-1 Box. (395 lbs.)

Containing 500 Crimson Rambler, Own Root.

62.50

Ring-1 Box (145 lbs.)

Containing 125 Eugene Marlatt, Own Root.

Ripe-1 Box (220 lbs.)

Containing 190 Red Radiance, Grafted.

\section{ROSES, NUMBER THREE GRADE-IN ROCHESTER}

\section{Transplanting Stock.}

Roast-1 Box (165 lbs.).

Containing 1225 Assorted, Own Root, as follows:

200 American Pillar

375 Philadelphia Rambler

100 White Rambler

200 Crimson Rambler

350 Veilchenblau

Robe-1 Box (110 lbs.)

Containing 850 Seven Sisters, Own Root.

Robust-1 Box (210 lbs.).

Containing 1400 Sir Thomas Lipton, Own Root.

Connecticut, Jan. 2nd, 1926: "Car FGE 33237 came through in fine shape. Everything has passed muster and bill is o.k.'d for June 1st."

West Va., March 8th, 1926: "The Barberry reached us in fine shape, the best we ever bought. Inclosed find our check."

Kentucky, March 4th, 1926: "Your car arrived March 3rd and unloaded same night. The stock is all in good condition and we feel that you are giving us a square deal in every way for which we are grateful and want to say "thank you'." 
 \\ CODE WORD \\ SHRUBS-IN ROCHESTER}

Shad-1 Box (565 lbs.)

PER BOX

$\$ 71.00$

Containing 1190 Assorted Shrubs, 2 yr., 12-18 in., well branched, as follows:

200 Hydrangea P. G.

125 Lonicera Morrowi

120 Spirea opulifolia aurea

575 Spirea VanHoutte

20 Viburnum Dentatum

150 Weigela Variegated

Shaft-1 Box (150 lbs.)

25.00

Containing 395 Assorted Althea, as follows:

325 Coelestis (Single Blue), 2 yr., 12-18 in., well branched

70 Rubis (Single Red), 2 yr., 18-24 in., well branched

Shake-1 Box (420 lbs.).

Containing 1020 Assorted Althea, as follows:

75 Boule de Feu, Tree Form, 18-24 in.

210 Boule de Feu, Bush, 2 yr., 18-24 in., well branched

575 Boule de Feu, Bush, 12-18 in., 2 yr., well branched

130 D'ble Purple Bush, 2 yr., 12-18 in., well branched

10 W. R. Smith (Single White), 12-18 in., 2 yr., well branched

20 W. R. Smith (Single White), 18-24 in., 2 yr., well branched

Sham-1 Box (110 lbs.)

Containing 440 Althea, 6-12 in., for transplanting, as follows:
50 Boule de Feu
290 Rubis
100 Purpurea semi plena

Shape-1 Box (160 lbs.)

Containing 140 Calycanthus, Assorted grades, as follows:

$60 \quad 2-3 \mathrm{ft}$., well branched $80 \quad 18-24$ in., well branched

Shark-2 Boxes (400 lbs.).

Each containing 300 Philadelphus Grandiflorus, $2-3$ ft., 2 yr., well branched.

Shave-2 Boxes (575 lbs.)

Each containing 230 Spirea Arguta, 30-36 in., well branched.

Shawl-1 Box (490 lbs.)

Containing 440 Spirea Arguta, 18-24 in., well branched.

Shed-1 Box (450 lbs.)

Containing 1500 Spirea VanHoutte, 2 yr., 12-18 in., well branched.

22.00

38.00

\section{PRIVET-IN ROCHESTER}

Hide-1 Box (560 lbs.)

Containing 490 Ibolium, 2 yr., 2 -3 ft., 4 or more canes.

Hint-1 Box (410 lbs.)

Containing 500 Ibolium, 2 yr., 18-24 in., 4 or more canes.

Hitch-1 Box (245 lbs.)

Containing 275 Ibolium, Assorted grades, as follows:

200 2-3 ft., 2 and 3 canes.

75 18-24 in., 2 and 3 canes.

Hive-1 Box (250 lbs.)

Containing 525 lbolium, $12-18$ in., 2 yr., 3 canes or more. 
CODE WORD

\section{VINES-IN ROCHESTER}

PRICE

PER BOX

Chafe-1 Box (135 lbs.) \$ 25.00

Containing 200 Ampelopsis Engelmanni, No. 1.

Chain-1 Box (325 lbs.)

Containing 1100 Ampelopsis Engelmanni, Medium.

Chalk-2 Boxes (195 lbs.)

Each containing 1250 Ampelopsis Engelmanni, No. 2.

Chance-1 Box (285 lbs.).

Containing 390 Ampelopsis Quinquefolia, No. 1.

Chapel-1 Box (350 lbs.)

Containing 950 Ampelopsis Quinquefolia, Medium.

Chart-1 Box (135 lbs.)

Containing 190 Honeysuckle, No. 1, as follows: 100 Halls Japan

90 Scarlet Trumpet

Cheese-1 Box (120 lbs.) Containing 60 White Wistaria, Grafted, 2 year XX.

Chest-1 Box (110 lbs.)

Containing 100 White Wistaria, Grafted, 2 year No. 1.

Cinder-2 Boxes (135 lbs.)

Each containing 150 White Wistaria, 1 Yr., Grafted No. 1.

Circle-1 Box (120 lbs.)

Containing 300 White Wistaria, 1 yr., grafted, Medium.

Civil-1 Box (75 lbs.).

Containing 150 White Wistaria, 1 yr., Grafted No. 2.

\section{NUT TREES-IN ROCHESTER}

Nail-2 Boxes (245 lbs.)

Each containing 50 English Walnut, 3-4 $\mathrm{ft}$.

Naked-1 Box (200 lbs.).

Containing 95 English Walnut, Assorted grades, as follows:

$25 \quad 2-3 \mathrm{ft}$.

50 18-24 in.

$20 \quad 12-18$ in.

30.00

30.00

\section{CLEMATIS}

Grown on contract near Rochester. Quantities to suit purchaser. Cost of packing to be added.

Price

Per 100

360 Henryii, 2 year Medium

$\$ 30.00$

240 Jackmanni, 2 year No. 1

45.00

520 Jackmanni, 2 year Medium.................................................... 30.00

470 Madam Edouard Andre, 2 year Medium.................................................... 30.00

200 Paniculata, 2 year No. 1 .

20.00

300 Paniculata, 2 year Medium.

15.00

Orders For Any of the Above Should Be Addressed To

\section{ROBERT C. CHASE}

Hotel Seneca

ROCHESTER, NEW YORK

PAGE 7 


\section{Send Orders For The Following To: \\ CHASE NURSERY COMPANY}

CHASE, ALABAMA

We are still filling orders for our friends in the South and are carrying in our cellars a fair assortment deciduous shrubs, roses, etc., but as we are near the end of the season many items in our list are exhausted. Glad to take care of you so far as we can, but suggest you give us as much leeway as possible in the matter of grades. Here's a special list of a few items we are not sending to storage. Price at Chase, packing extra.

\section{DECIDUOUS SHRUBS}

50 Baccharis halimifolia, 3-4 $\mathrm{ft}$

PRICE EACH

400 Jasminum nudiflorum, 2-3 $\mathrm{ft}$.

\$ .30

600 Jasminum nudiflorum, 18-24 in............................................................. .15

800 Jasminum nudiflorum, $12-18$ in...........................................................

900 Lonicera Fragrantissima, 3-4 ft...........................................................

870 Lonicera Fragrantissima, 2-3 ft........................................................... $\quad .20$

2 Magnolia Alba superba, 3-4 ft., B\&B................................................... 7.50

15 Magnolia Alba superba, 2-3 ft., B\&B.................................................... 6.00

3 Magnolia Alba superba, 18-24 in., B\&B.............................................. 4.50

6 Magnolia Alexandrina, 18-24 in., B\&B................................................ 4.50

6 Magnolia Alexandrina, 12-18 in., B\&B................................................. 3.00

2 Magnolia Lenne, 2-3 ft., B\&B.......................................................... 6.00

3 Magnolia Lenne, 18-24 in., B\&B...................................................... 4.50

2 Magnolia Soulangeana, 18-24 in., B\&B............................................... 4.50

3500 Privet California, 3-4 ft., 2 and 3 canes............................................. . .05

2100 Privet California, 2-3 ft., 4 or more canes.............................................. . .05

50 Vitex Agnus castus, 3-4 ft.................................................................. .25

140 Vitex Agnus castus, 2-3 ft....................................................................

60 Vitex Agnus castus, 18-24 in................................................................... .15

\section{EVERGREEN SHRUBS}

500 Abelia grandiflora, 3-4 ft., B\&B........................................................ 1.00

600 Abelia grandiflora, 2-3 ft., Puddled................................................... .35

1500 Abelia grandiflora, 18-24 in., Puddled................................................... .25

100 Euonymus Bungeanus, 2-3 ft., Puddled............................................... .40

200 Euonymus Bungeanus, 18-24 in., Puddled............................................... $\quad .30$

100 Euonymus Bungeanus, $12-18$ in., Puddled...………................................ $\quad .20$

300 Euonymus Radicans, 15-18 in., Puddled.................................................... .25

500 Euonymus Radicans, $12-15$ in., Puddled.................................................... $\quad .20$

500 Euonymus Radicans, 8-12 in., Puddled.................................................... $\quad .10$ 


\section{STOCK IN CELLARS AT CHASE-Continued}

150 Gardenia Florida, 18-24 in Puddled

300 Gardenia Florida, 15-18 in., Puddled

200 Gardenia Florida, 12-15 in., Puddled......................................................... . 30

150 Laurocerasus Caroliniana, 3-4 ft., B\&B............................................ 1.25

220 Laurocerasus Caroliniana, 2-3 ft., B\&B.................................................. $\quad .75$

300 Ligustrum Japonicum, 3-4 ft., Puddled.................................................. . .50

600 Ligustrum Japonicum, 2-3 ft., Puddled.................................................. .40

200 Ligustrum Japonicum, 18-24 in., Puddled........................................... . .30

50 Ligustrum Lucidum, 2-3 ft., B\&B....................................................... 1.15

200 Ligustrum Lucidum, 18-24 in., B\&B.................................................. .95

\section{CONIFERS}

150 Cedrus deodara, 30-36 in., B\&B....................................................... 3.50

150 Cedrus deodara, 24-30 in., B\&B......................................................... 2.75

100 Juniperus Communis, 30-36 in., B\&B.................................................... 1.00

50 Thuya aurea nana (Berckman's golden), 24-30 in., B\&B................... 3.00

200 Thuya aurea nana (Berckman's golden), 18-24 in., B\&B................... 2.50

200 Thuya aurea nana (Berckman's golden), 15-18 in., B\&B................... 1.75

- New Jersey, March 2nd, 1926: "Shipment arrived in good condition and enclosed find my check, \$197.23, to cover."

Georgia, Feb. 22nd, 1926: "The Abelias received and as we expected were up to the Chase standard, they were mighty nice."

Illinois, Jan. 9th, 1926: "Your Amoor River North and Spirea VanHoutti are exceptionally nice, root system fine."

Mississippi, Feb. 20th, 1926: "Want to thank you for the fine lot of Abelia plants sent us in December, every one is living."

New Jersey, Jan. 29th, 1926: "Pleased to advise that the second car from you has come in and though I am still pleading with our foreman for an unfavorable report covering the stock in this car, 1 haven't as yet made the slightest headway."

Kentutcky, Jan. 9th, 1926: "Check herewith, $\$ 108.70$, to cover your invoices. Stock nice, well pleased. Would like to get some more of the Spruce."

Tennessee, Nov. 30th, 1925: "Everything from you satisfactory as usual. Check herewith to balance."

Georgia, Nov. 23rd, 1925: "Shrubs arrived, and both packing and quality of plants all we anticipated. Check herewith to cover. 
